\title{
Tamsulosin plus a new complementary and alternative medicine in patients with lower urinary tract symptoms suggestive of benign prostatic hyperplasia: Results from a retrospective comparative study
}

\author{
Ferdinando Fusco, Massimiliano Creta, Francesco Trama, Fabio Esposito, Felice Crocetto, \\ Achille Aveta, Francesco Mangiapia, Ciro Imbimbo, Marco Capece, Roberto La Rocca, \\ Vincenzo Mirone, Nicola Longo
}

Department of Neurosciences, Reproductive Sciences and Odontostomatology, University of Naples Federico II. Naples, Italy.

\begin{abstract}
Summary Background: We aimed to compare the efficacy of tamsulosin $0.4 \mathrm{mg}$ once a day alone and the combination therapy involving tamsulosin $0.4 \mathrm{mg}$ once a day plus the complementary and alternative medicine consisting of vitamins (C and D), herbal products (Cucurbita maxima, Capsicum annum, Polygonum capsicatum) and amino acid L-Glutamine bid in patients with lower urinary tract symptoms related to benign prostatic hyperplasia (LUTS/BPH). Methods: We performed a retrospective matched paired comparison. The clinical records of LUTS/BPH patients who underwent medical therapy with tamsulosin $0.4 \mathrm{mg} /$ day plus the complementary and alternative medicine consisting of vitamins (C and D), herbal products (Cucurbita maxima, Capsicum annum, Polygonum capsicatum) and amino acid L-Glutamine bid between January 2019 to September 2019 were reviewed (Group 1). These patients were compared in a 1:1 fashion with LUTS/BPH patients who underwent therapy with tamsulosin $0.4 \mathrm{mg} /$ day alone (Group 2). Total, storage, voiding and Quality of Life (QoL) international prostate symptom (IPSS) score, as well as overactive bladder (OAB)-v8 score and treatment-related adverse events recorded at 40 days follow-up in both groups were compared.

Results: At 40 days follow-up mean total, storage, voiding and QoL IPSS sub-scores as well as OAB-v8 score significantly improved in both groups. Intergroup comparison showed statistically significant lower mean total IPSS score (11.6 vs 12.4, $p=0.04$ ) mean storage IPSS sub-score (6.5 vs 7.5, $p=0.01$ ), and mean $O A B$ v8 score (16.7 vs $18.8, p=0.03)$ in patients in the Group 1.

Conclusions: The combination of tamsulosin $0.4 \mathrm{mg} /$ die plus the complementary and alternative medicine consisting of vitamins (C and D), herbal products (Cucurbita maxima, Capsicum annum, Polygonum capsicatum) and amino acid LGlutamine bid provides statistically significant advantages in terms of storage LUTS improvements in patients with LUTS/BPH compared to tamsulosin $0.4 \mathrm{mg} /$ day alone. These findings are preliminary and further prospective studies on a greater number of patients are needed to confirm it.
\end{abstract}

KEY WORDS: Benign prostatic hyperplasia; Combination therapy; Lower urinary tract symptoms; Phytotherapy.

Submitted 20 February 2020; Accepted 13 March 2020

\section{INTRODUCTION}

Lower urinary tract symptoms related to benign prostatic hyperplasia (LUTS/BPH) represent a common complaint in everyday urological practice and their prevalence increases with ageing (1-3). In the EPIC study, Riboli et al. reported an incidence of storage and voiding LUTS of about 51\% and $26 \%$ of men evaluated, respectively (3). Interestingly, approximately $18 \%$ of men reported the coexistence of storage and voiding symptoms $(4,5)$. The European Association of Urology (EAU) guidelines strongly recommend $\alpha 1$-adrenoceptor antagonists as first-line therapeutic option in patients with moderate to severe symptoms as they significantly improve urinary symptoms and maxim urinary flow $\left(\mathrm{Q}_{\max }\right)(6)$. In men with moderate-to-severe LUTS who mainly have bladder storage symptoms EAU Guidelines strongly recommend muscarinic receptor antagonists (strong recommendation) or beta-3 agonists (weak recommendation) (7). However, a number of concerns have been reported with the prescription of these drugs. Antimuscarinics might theoretically decrease bladder strength, thus increasing post-void residual volume (PVR) urine and causing urinary retention. Moreover, not all antimuscarinics have been evaluated in elderly men, and long-term studies on their efficacy in men of any age with LUTS are not yet available. Furthermore, antimuscarinics are contraindicated in patients with angle-closure glaucoma, gastrointestinal obstruction, paralytic ileus, myasthenia gravis, severe heart disease $(8,9)$. On the other hand, mirabegron has been evaluated mainly in female patients $(8,9)$. In recent years the prescription of phytotherapeutic compounds in patients with LUTS/BPH has gained growing interest (10). These agents represent a heterogeneous group and may contain differing concentrations of active ingredients. The complementary and alternative medicine Kubiker (Naturmed, Italy), consisting of vitamins (C and D), herbal products (Cucurbita maxima, Capsicum annum, Polygonum capsicatum) and amino acid LGlutamine, has been proposed in the treatment of overactive bladder syndrome (OAB) (11). We aimed to compare the efficacy of the combination therapy involving tamsulosin $0.4 \mathrm{mg}$ once a day plus Kubiker bid and therapy with tamsulosin $0.4 \mathrm{mg}$ alone in patients with LUTS/BPH.

\section{MATERIALS AND METHOdS}

We performed a retrospective comparative study. The clinical records of LUTS/BPH patients who underwent 
medical therapy with tamsulosin $0.4 \mathrm{mg}$ /day plus Kubiker bid between January 2019 to September 2019 were reviewed (Group 1). These patients were compared in a 1:1 fashion with LUTS/BPH patients who underwent therapy with tamsulosin $0.4 \mathrm{mg}$ /day alone (Group 2). The followings were considered exclusion criteria: post-void residual volume $(\mathrm{PVR})>150 \mathrm{ml}$, prostate specific antigen (PSA) > $10 \mathrm{ng} / \mathrm{ml}$, concomitant therapy with 5-alpha reductase inhibitors and/or phosphodiesterase type 5 inhibitors and/or muscarinic receptor antagonists or beta3 agonists, presence of neurological disorders, previous pelvic surgery, diabetes, urinary tract infections, history of acute urinary retention. The matched-pair comparison was based on the following criteria: PSA, prostate volume (PV), $\mathrm{Q}_{\max }$, PVR, total international prostate symptom score (IPSS), and 8-item overactive bladder questionnaire 8 (OAB-v8) score. Total, storage, voiding and Quality of Life (QoL) IPSS scores, as well as OAB-v8 score and treatment-related adverse events recorded at 40 days followup in both groups were compared. Descriptive data of continuous variables were expressed as mean \pm standard deviation (SD) and compared using the Student's t tests. The analyses were considered significant for a p-value $<0.05$. All statistical analyses were performed with SPSS version 16.0 software.

The study was performed in accordance with the ethical standards laid down in the Declaration of Helsinki. Verbal informed consent was obtained from subjects.

\section{RESULTS}

Overall, 36 eligible patients who underwent medical therapy with tamsulosin $0.4 \mathrm{mg} /$ day plus Kubiker were identified and compared to 36 patients who underwent therapy with tamsulosin $0.4 \mathrm{mg} /$ day alone. Baseline patients' characteristics in both groups are reported in Table 1. At 40 days follow-up mean total, storage, voiding and QoL IPSS sub-scores significantly improved in both groups (Table 2). Similarly, a statistically significant improvement in terms of OAB v8 score and $Q_{\max }$ was observed in both groups (Table 2).

Intergroup comparison showed statistically significant lower mean total IPSS score, mean storage IPSS sub-score,

\section{Table 1.}

Baseline patients' characteristics in both groups.

\begin{tabular}{|c|c|c|c|}
\hline & Group $1(n=36)$ & Group $2(n=36)$ & p \\
\hline Age, years, mean (SD) & $65.3(9.6)$ & $63.4(8.5)$ & 0.32 \\
\hline Prostate volume, mL, mean (SD) & $44.1(24.4)$ & $46.1(22.7)$ & 0.73 \\
\hline PSA, ng/mL, mean (SD) & $2.9(0.8)$ & $3.2(0.4)$ & 0.91 \\
\hline PVR, mL, mean (SD) & $37.0(11.2)$ & $39.0(10.8)$ & 0.26 \\
\hline Qmax, mL/sec, mean (SD) & $11.2(0.7)$ & $12.5(0.8)$ & 0.18 \\
\hline IPSS total, mean (SD) & $17.9(0.9)$ & $18.0(0.8)$ & 0.59 \\
\hline IPSS voiding, mean (SD) & $8.5(2.2)$ & $9.1(1.3)$ & 0.15 \\
\hline IPSS storage, mean (SD) & $9.3(2.0)$ & $8.8(1.2)$ & 0.18 \\
\hline IPSS QoL, mean (SD) & $2.9(0.3)$ & $3.2(0.4)$ & 0.23 \\
\hline OAB v8, mean (SD) & $19.6(0.8)$ & $20.1(0.9)$ & 0.29 \\
\hline
\end{tabular}

IPSS: International Prostate Symptom Score; QoL: Quality of Life; OAB v8: 8-item overactive bladder questionnaire; PSA: Prostate Specific Antigen; PVR: Post-void residua volume; $Q_{\text {max }}$ maximum urinary flow; SD: Standard Deviation.
Table 2.

IPSS and OAB v8 scores in both groups at 40-day follow-up.

\begin{tabular}{|c|c|c|c|}
\hline & Group $1(n=36)$ & Group 2 ( $n=36$ ) & $p$ \\
\hline IPSS total, mean (SD) & $11.6(1.7)^{*}$ & $12.4(1.5)^{*}$ & 0.04 \\
\hline IPSS voiding, mean (SD) & $5.1(2.1)$ & $4.8(1.1)$ & 0.58 \\
\hline IPSS storage, mean (SD) & $6.5(1.9)^{*}$ & $7.5(1.6)^{*}$ & 0.01 \\
\hline IPSS QoL, mean (SD) & $2.1(0.8)$ & $2.4(0.8)$ & 0.20 \\
\hline$\overline{O A B}$ v8, mean (SD) & $16.7(0.5)^{*}$ & $18.8(0.8)^{*}$ & 0.03 \\
\hline$Q_{\text {max }} \mathrm{mL} / \mathrm{sec}$, mean (SD) & $13.6(0.8)$ & $14.1(0.7)$ & 0.20 \\
\hline$\overline{P V R}, \mathrm{~mL}$, mean (SD) & $28.0(10.2)$ & $32.0(8.8)$ & 0.42 \\
\hline \multicolumn{4}{|c|}{$\begin{array}{l}\text { IPSS: International Prostate Symptom Score; OAB v8: 8-item overactive bladder questionnaire; } \\
\text { PVR: Post-void residua volume; } Q_{\max } \text { maximum urinary flow; QoL: Quality of Life; SD: Standard Deviation. } \\
*: \text { : }<0.05 \text { with respect to baseline. }\end{array}$} \\
\hline
\end{tabular}

and mean $\mathrm{OAB}$ v8 scores in patients in the Group 1. Not statistically significant differences in terms of voiding IPSS sub-score, $\mathrm{Q}_{\max }$ and PVR emerged from intergroup analysis. Not clinically significant treatment-related adverse events were recorded in both groups.

\section{Discussion}

Benign prostatic obstruction has been reported to cause morpho-functional alterations involving the detrusor muscle. Clinically, these alterations can impair bladder contractility and cause detrusor overactivity, decreasing bladder compliance, and onset of storage LUTS characterized by an altered bladder sensation, increased daytime frequency, nocturia, urgency and urgency incontinence (121). Experimental models have shown that bladder outlet obstruction causes detrusor smooth muscle cells hypertrophy and hyperplasia as well as extracellular matrix alterations that may lead, over time, to detrusor overactivity and, later, to reduced bladder contractility (13-15). As reported in the EpiLUTS study, $45.7 \%$ of the 14.139 men evaluated had storage LUTS (16). $\alpha 1$-blockers act by inhibiting the effect of endogenously released noradrenaline on smooth muscle cells in the prostate thus reducing prostate tone and bladder outlet obstruction (17). These drugs can reduce both storage and voiding LUTS and are considered the first-line drug treatment for male LUTS due to their good efficacy, and low rate and severity of adverse events. LUTS/BPH patients with mainly bladder storage symptoms represent a difficult to treat subset of patients. Indeed, therapy with $\alpha 1$-blockers may be suboptimal. On the other hand, both muscarinic receptor antagonists and beta-3 agonists should be prescribed with cautions and adherence to treatments with these drugs is often inadequate. Herbal treatments are an increasingly popular alternative for treating storage LUTS (18). To the best of our knowledge, we compared, for the first time, the clinical efficacy of the combination of tamsulosin $0.4 \mathrm{mg} /$ day plus Kubiker and tamsulosin $0.4 \mathrm{mg} /$ day alone in patients with LUTS/BPH. We found that the combination therapy provided statistically significant advantages in terms of storage LUTS as demonstrated by lower IPSS storage sub-scores as well as lower OAB-v8 score. A number of evidences exist about the potential beneficial effects provided by the compounds contained in the food supple- 
ment Kubiker. Cucurbita maxima, contained in pumpkin seeds, has been reported to provide benefits in both preclinical and clinical models of lower urinary tract dysfunction (19-25). Pre-clinical studies have shown that pumpkin seeds have antioxidant and inflammatory properties and inhibit lipid peroxidation (20). Pumpkin seeds administered to rats affected by overactive bladder (OAB) syndrome showed to cause an increase of the production of nitric oxide (NO) via the NO/arginine pathway (22). Independently of the acetylcholin/adrenaline system, this pathway generated the relaxation of the bladder detrusorial musculature (23). Pumpkin seeds were also shown to modulate prostate growth. Abdel Rahman et al. found that rats fed with high amounts of pumpkin seeds in the diet had smaller prostate sizes as compared to untreated rats (24). Furthermore, Tsai and co-workers showed that rats receiving subcutaneous testosterone to induce an increase in prostate size and subsequently treated with pumpkin seeds for 14 days, presented a smaller prostate gland compared to the control group treated only with prazosin (25). Nishimura et al. observed that the administration of pumpkin seed extract for 12 weeks significantly reduced the symptoms of OAB with no side effects (19). Polygonum capsicatum has a strong antioxidant activity, which has been observed in vitro (26). Capsaicin is the first vanilloid investigated for therapeutic purposes and evidence exists demonstrating its efficacy in the treatment of LUTS (11). The capsaicin has been used for the treatment of $O A B$ syndrome due to its ability to desensitize the transient receptor potential vanilloid 1 receptor (27). Evidence exists demonstrating that vitamin $C$ from food and beverages can modulate voiding symptoms (11). However, the knowledge of the exact mechanism of action deserves further investigations. Vitamin D is essential for the proper functioning of the pelvic floor. It has been widely reported that a vitamin deficiency can predispose patients to a high risk of developing LUTS and incontinence $(28,29)$. To date, the role of glutamine in patients with LUTS is widely under-investigated and deserves careful investigations. Overall, although preliminary, results from the present study have relevant clinical implications and pose the basis for further investigations. The combination of $\alpha 1$-adrenoceptor antagonists and phytotherapeutic agents containing a mixture of compounds that can interfere with the pathophysiology of bladder dysfunction at multiple levels like Kubiker may represent a strategy to discuss in patients with prevalent storage LUTS/BPH for which therapy with $\alpha 1$-adrenoceptor antagonists alone is suboptimal and medical treatments with muscarinic receptor antagonists or beta-3 agonists are not recommended or not tolerated.

The main limits of the present study are the retrospective design, the small sample size, and the short follow-up. Moreover, the specific role of the various components of Kubiker could not be assessed. Therefore, results from the present study should be considered preliminary and further studied are needed to confirm the efficacy and safety of the combination of tamsulosin and the food supplement Kubiker in LUTS/BPH patients and to identify the subset of patients that can benefit most from this approach.
The role of Kubiker in women with storage LUTS represents a further area of interest $(11,30)$.

\section{Conclusions}

The combination of tamsulosin $0.4 \mathrm{mg}$ /die plus Kubiker bid provides statistically significant advantages in terms of storage LUTS improvements in patients with LUTS/BPH compared to tamsulosin $0.4 \mathrm{mg}$ /day alone. These findings are preliminary and further prospective studies on a greater number of patients are needed to confirm it.

\section{REFERENCES}

1. Mirone V, Carrieri G, Morgia G, et al. Risk factors for benign prostatic enlargement: The role of lifestyle habits at younger age. The \#Controllati2017 initiative study group. Arch Ital Urol Androl. 2017; 89:253-258.

2. Irwin DE, Milsom I, Kopp Z, et al. Prevalence, severity, and symptom bother of lower urinary tract symptoms among men in the EPIC study: impact of overactive bladder. Eur Urol. 2009; 56: 14-20.

3. Riboli E, Hunt KJ, Slimani $N$, et al. European Prospective Investigation into Cancer and Nutrition (EPIC): study populations and data collection. Public Health Nutr. 2002; 5:1113-1124.

4. Witjes WP, de la Rosette JJ, Donovan JL, et al. The International Continence Society "Benign Prostatic Hyperplasia" Study: international differences in lower urinary tract symptoms and related bother. J Urol. 1997; 157:1295-300

5. Chung DE, Sandhu JS. Overactive bladder and outlet obstruction in men. Curr Urol Rep. 2011; 12:77-85.

6. Kortmann BB, et al. Urodynamic effects of alpha-adrenoceptor blockers: a review of clinical trials. Urology. 2003; 62:1.

7. Chapple CR, et al. A shifted paradigm for the further understanding, evaluation, and treatment of lower urinary tract symptoms in men: focus on the bladder. Eur Urol. 2006; 49:651.

8. Yamaguchi O, Marui E, Igawa Y, et al. Efficacy and safety of the selective $\beta 3$-adrenoceptor agonist mirabegron in Japanese patients with Overactive Bladder: a randomized, double-blind, placebocontrolled, dose-finding study. Low Urin Tract Symptoms. 2015; $7: 84-92$.

9. Oelke M, Bachmann A, Descazeaud A, et al. EAU guidelines on the treatment and follow-up of non-neurogenic male lower urinary tract symptoms including benign prostatic obstruction. Eur Urol. 2013; 64:118-40

10. Cicero AFG, Allkanjari O, Busetto GM, et al. Nutraceutical treatment and prevention of benign prostatic hyperplasia and prostate cancer.Arch Ital Urol Androl.. 2019; 91:139-152.

11. Vecchioli-Scaldazza C, Morosetti C, Maruccia S, et al. Randomized, multicenter, controlled study, comparing efficacy and safety of a new complementary and alternative medicine (CAM) versus Solifenacin Succinate in women with overactive bladder syndrome. Arch Ital Urol Androl. 2017; 89:296-300.

12. Fusco F, Creta M, Imperatore V, et al. Benign prostatic obstruction relief in patients with lower urinary tract symptoms suggestive of benign prostatic enlargement undergoing endoscopic surgical procedures or therapy with alpha-blockers: review of urodynamic studies. Adv Ther. 2017; 34:773-783.

13. Levin RM, Monson FC, Haugaard N, et al. Genetic and cellular characteristics of bladder outlet obstruction. Urol Clin North Am. 1995; 22:263-83 
14. Fusco F, Creta M, De Nunzio C, et al. Progressive bladder remodeling due to bladder outlet obstruction: a systematic review of morphological and molecular evidences in humans. BMC Urol. 2018; 18:15

15. Gravas S, Cornu JN, Gacci M. Management of Non-Neurogenic Male Lower Urinary Tract Symptoms (LUTS), incl. Benign Prostatic Obstruction (BPO). EAU guidelines, 2019.

16. Sexton CC, Coyne KS, Kopp ZS, et al. The overlap of storage, voiding and postmicturition symptoms and implications for treatment seeking in the USA, UK and Sweden: EpiLUTS. BJU Int 2009; 103(Suppl. 3):12-23.

17. Creta M, Bottone F, Sannino S, et al. Effects of alphal-blockers on urodynamic parameters of bladder outlet obstruction in patients with lower urinary tract symptoms suggestive of benign prostatic enlargement: a review. Minerva Urol Nefrol 2015 Oct 27.

18. Chughtai B, Kavaler E, Lee R, et al. Use of herbal supplements for overactive bladder. Rev Urol. 2013; 15:93-6.

19. Nishimura M, Ohkawara T, Sato H, et alPumpkin seed oil extracted from Cucurbita maxima improves urinary disorder in human overactive bladder. J Tradit Complement Med. 2014; 4:72-74.

20. Xanthopoulou MN, Nomikos T, Fragopoulou E, Antioxidant and lipoxygenase inhibitory activities of pumpkin seed extracts. Food Res Int. 2009; 42:641-646.

21. Fahim AT, Abd-el-Fattah AA, Agha AM, Effect of pumpkin-seed oil on the level of free radical scavengers induced dur-ing adjuvantarthritis in rats. Pharmacol Res. 1995; 31:73-79.

22. Hata K Tanahashi S, Wakida Y, Tatsuzaki M, Koide A. Effect of
Pumpkin seed extract on urinary bladder function in anesthetized rats. Jpn J Med Pharm Sci. 2005;5:339-45.

23. Andersson KE, Wein AJ. Pharmacology of the lower urinary tract: basis for current and future treatments of urinary incontinence. Pharmacol Rev. 2004; 56:581-63.

24. Abdel-Rahman MK. Effect of pumpkin seed (Cucurbita pepo L) diets on benign prostatic hyperplasia (BPH): chemical and morphometric evaluation in rats. World J Chem. 2006; 1:33-40.

25. Tsai Y-S, Tong Y-C, Cheng J-T, et al. Pumpkin seed oil and phytosterol-F can block testosterone/prazosin-induced prostate growth in rats. Urol Int. 2006; 77:269-274.

26. Kirino A, Takasuka Y, Nishi A, et al. Analysis and functionality of major polyphenolic components of Polygonum cuspidatum (itadori). J Nutr Sci Vitaminol. (Tokyo). 2012; 58:278-286.

27. Cruz F. Desensitization of bladder sensory fibers by intravesical capsaicin or capsaicin analogs. A new strategy for treatment of urge incontinence in patients with spinal detrusor hyperreflexia or bladder hypersensitivity disorders. Int Urogynecol J. 1998; 9:214-220.

28. Vaughan CP, Johnson TM, Goode PS, et al. Vitamin D and lower urinary tract symptoms among men: results from the 2005- 2006 National Health and Nutrition Examination Survey. Urology. 2011 78:1292-1297.

29. Ishazly MA, Sultan MF, Aboutaleb HA, et al. Vitamin D deficiency and lower urinary tract symptoms in males above 50 years of age. Urol Ann. 2017; 9:170-173.

30. Xu P, Yong Q. Female urinary incontinence: diagnosis and treatment. Journal of Genitourinary Research and Practice. 2019; 1:22-25.

\section{Correspondence}

Ferdinando Fusco, MD

ferdinando-fusco@libero.it

Massimiliano Creta, MD

max.creta@gmail.com

Francesco Trama, MD (Corresponding Author)

francescotrama@gmail.com

Fabio Esposito, MD

fabioesposito025@gmail.com

Felice Crocetto, MD

felice.crocetto@gmail.com

Achille Aveta, MD

achille-aveta@hotmail.it

Francesco Mangiapia, MD

mangiapippo@libero.it

Ciro Imbimbo, MD

imbimbo@unina.it

Marco Capece, MD

Roberto La Rocca, MD

robertolarocca87@gmail.com

Vincenzo Mirone, MD

mirone@unina.it

Nicola Longo, MD

Department of Neurosciences, Reproductive Sciences and Odontostomatology,

University of Naples Federico II

Via Sergio Pansini 5, Napoli (Italy) 